\title{
Breakthrough: PAK1-dependent expression of PD-L1 (programmed death ligand)
}

\author{
Hiroshi Maruta* \\ PAK Research Center, Melbourne, 3055, Australia
}

\begin{abstract}
The "Nobel-winning" monoclonal antibodies against programmed death ligand (PD-L1) or its receptor (PD-1) have been used clinically for immuno (check point) therapy of cancers such as melanomas and lung cancers, but not Gemcitabine (GEM)-resistant pancreatic cancers. However, monoclonals are very expensive and are unable to pass BBB (blood brain barrier). Thus, we have been developing a series of alternative (inexpensive) chemical "check point" therapeutics of small molecular weight. Some of them are among potent (highly cell-permeable and water-soluble) PAK1-blockers such as 15K (1,2,3-triazolyl ester of Ketorolac) and Frondoside A (FRA) from sea cucumber. PAK1 (RAC/CDC42-activated kinase 1) is the major oncogenic/ageing Ser/Thr kinase which is essential for both growth and metastasis of solid tumors as well as responsible for shortening the healthy lifespan as well. The major reason behind this project is based on the following hypothesis: Expression of PD-L1 depends on the oncogenic RAS-JAK-PAK1 signaling pathway. In fact, genetic silencing of PAK1 suppresses PD-L1 expression in human pancreatic cancers. Thus, it is most likely that the potent PAK1-blockers such as 15K and FRA would be inexpensive "chemical" check point thera-peutics for cancers including GEM-resistant pancreatic cancers. We are currently confirming this "working” hypothesis with 15K, FRA and a few other PAK1-blockers.
\end{abstract}

\section{Background behind the hypothesis}

The pioneer work on monoclonal-based immune (check-point) therapy of cancers by Tasuku Honjo and Jim Allison was highly recognized by 2018 Nobel Prize in physiology/ medicine. Since the check-point ligands such as PD-L1 (programmed death ligand) and its receptor PD-1 on cancers are responsible for their avoidance of so-called "immune surveillance" by T-cells, monoclonal antibodies against these progammed death ligands or receptors would be useful for therapy of cancers in particular melanomas and lung cancers which express these ligands or receptors at high levels $[1,2]$. However, so far, these monoclonals have not be successful clinically against pancreatic cancers which carry the oncogenic RAS mutant, but express PD-L1 only at low levels. $90 \%$ of pancreatic cancers are resistant to Gemcitabine (GEM), and one of the reasons for their GEM-resistance was found that GEM promotes PD-L1 expression through a Tyr-kinase family called JAK, destroying anti-tumor T-cells [3].

Since the major oncogenic/ageing Ser/Thr kinase PAK1 is activated by Tyr-kinase family called JAKs directly, we wonder if PAK1 is also responsible for RAS-JAK induced expression of PD-L1 [4]. Interestingly, PAK1 is responsible for GEM-resistance, and also for promoting T/Bcell based immune response in mice [5,6]. Thus, in theory, it is quite possible that RAS/JAK-dependent PD-L1 expression requires PAK1 as well (Figure. 1). In fact, we recently confirmed that silencing PAK1 by Si RNA in pancreatic cancer cells down-regulates PD-L1 [7]. Furthermore, inhibition of MEK, a Tyr-kinase down-stream of PAK1, also downregulates $\mathrm{PD}-\mathrm{L} 1$ and is synergestic with anti-PD-L1 monoclonal to suppress the growth of colon cancers in vivo $[8,9]$.

Accordingly, we have reached the inescapable conclusion that anti-cancer PAK1-blockers (natural or synthetic) in general could suppress PD-L1 expression in cancers including GEM-resistant pancreatic cancers. In support of this conclusion, several distinct PAK1-blockers such as triptolide, resveratrol, curcumin, melatonin and AG490 have been reported to be among PD-L1 suppressors
[3,10-16]. As summarized in Figure 2, the radiation-induced PD-L1 expression in glioma cells is almost completely suppressed by AG490, a JAK inhibitor, that blocks the EGFR-RAS-JAK-PAK1-ERK signaling pathway [16]. Among the natural PAK1-blockers, so far triptolide is the most potent, suppressing the $\mathrm{PD}-\mathrm{L} 1$ expression with the $\mathrm{IC}_{50}$ around $30 \mathrm{nM}$ (11), by inhibiting directly both RAC and JAK, the two direct activators of PAK1 $[4,17,10]$.

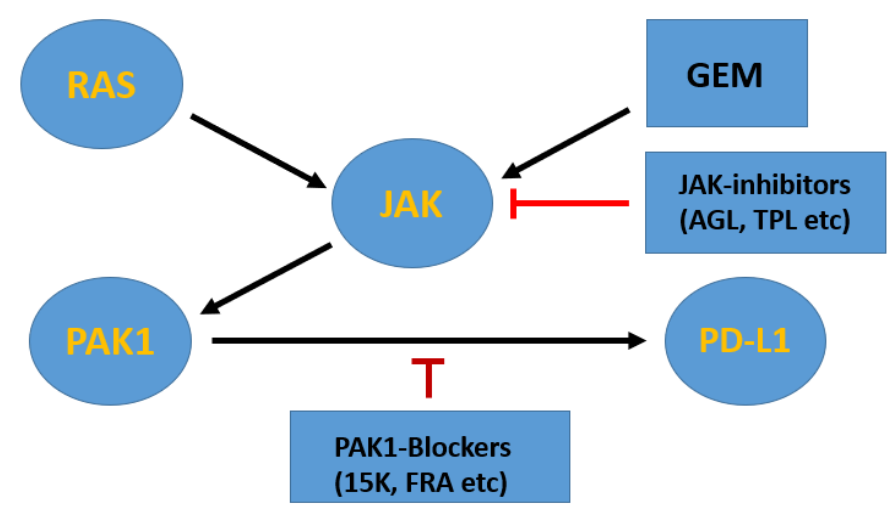

Figure 1. The RAS-PAK1 signalling pathway leading to PD-L1 expression In GEM-resistant pancreatic cancers which carry the oncogenic RAS mutant, PD-L1 gene is activated by both RAS and GEM through JAK-PAK1 signalling pathway. Thus, in theory, JAK inhibitors such as AGL (andrographolide) and TPL (triptolide) or PAK1-blockers such as $15 \mathrm{~K}$ and FRA (Frondoside A) could suppress PD-L1 expression

${ }^{*}$ Correspondence to: Hiroshi Maruta, PAK Research Center, Melbourne, 3055, Australia, E-mail: maruta20420@yahoo.co.jp

Key words: PAK1, PD-L1, cancer, check-point therapy, immuno-suppressor

Received: January 04, 2019; Accepted: January 16, 2019; Published: January 18, 2019 
U87

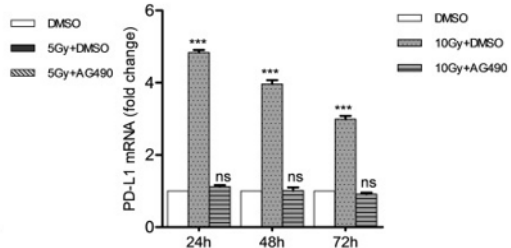

U251
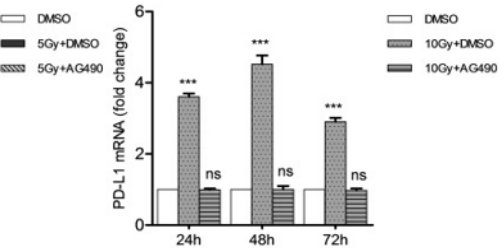

Figure 2. AG490 suppresses the radiotherapy (RT) -induced PD-L1 expression The PD-L1 expression (mRNA level) in glioma cell lines, which was boosted by increasing doses of RT (Gy), was almost completely suppressed by AG490 $(10 \mu \mathrm{M})$ that block the EGFR-RAS-JAK-PAK1-ERK signalling pathway by inhibiting JAK directly [16]

\section{Acknowledgement}

The author is very grateful to Dr. Hong He at Melbourne University Hospital for her unpublished information on PAK1-dependent PD-L1 expression.

\section{References}

1. Iwai Y, Ishida M, Tanaka Y, Okazaki T, Honjo T, et al. (2002) Involvement of PD-L1 on tumor cells in the escape from host immune system and tumor immunotherapy by PD-L1 blockade. Proc Natl Acad Sci U S A 99: 12293-12297. [Crossref]

2. Wei SC, Levine JH, Cogdill AP, Zhao Y, Anang NAS, et al (2017) Distinct cellular mechanisms underlie anti-CTLA-4 and anti-PD-1 checkpoint blockade. Cell 170: 1120-1133.

3. Doi T, Ishikawa T, Okayama T, Oka K, Mizushima K, et al. (2017) The JAK/STAT pathway is involved in the upregulation of PD-L1 expression in pancreatic cancer cell lines. Oncol Rep 37: 1545-1554. [Crossref]

4. Rider L, Shatrova A, Feener EP, Webb L, Diakonova M (2007) JAK2 tyrosine kinase phosphorylates PAK1 and regulates PAK1 activity and functions. J Biol Chem 282: 30985-30996. [Crossref]
5. Jagadeeshan S, Subramanian A, Tentu S, Beesetti S, Singhal M, et al. (2016) P21activated kinase 1 (Pak1) signaling influences therapeutic outcome in pancreatic cancer. Ann Oncol 27: 1546-1556.

6. Huynh N, Wang K, Yim M, Dumesny CJ, Sandrin MS, et al. (2017) Depletion of p21activated kinase 1 up-regulates the immune system of APCâ^ $\uparrow 14 /+$ mice and inhibits intestinal tumorigenesis. BMC Cancer 17: 431. [Crossref]

7. He H (2019) A genetic evidence for PAK1-driven expression of PD-L1 in pancreatic cancers. Personal Communication.

8. Jiang X, Zhou J, Giobbie-Hurder A, Wargo J, Hodi FS (2013) The activation of MAPK in melanoma cells resistant to BRAF inhibition promotes PD-L1 expression that is reversible by MEK and PI3K inhibition. Clin Cancer Res 19: 598-609.

9. Ebert PJR, Cheung J, Yang Y, McNamara E, Hong R, et al. (2016) MAP kinase inhibition promotes $\mathrm{T}$ cell and anti-tumor activity in combination with PD-L1 checkpoint blockade. Immunity 44: 609-621. [Crossref]

10. Maruta H, Ahn MR (2017) From bench (laboratory) to bed (hospital/home): How to explore effective natural and synthetic PAK1-blockers/longevity-promoters for cancer therapy. Eur J Med Chem 142: 229-243.

11. Liang M, Fu J (2008) Triptolide inhibits interferon-gamma-induced programmed death-1-ligand 1 (PD-L1) surface expression in breast cancer cells. Cancer Lett 270: 337-341.

12. Chin YT, Wei PL (2018) Thyroxine inhibits resveratrol-caused apoptosis by PD-L1 in ovarian cancer cells. Endocr Relat Cancer 25: 533-545. [Crossref]

13. Liao F, Liu L, Luo E, Hu J (2018) Curcumin enhances anti-tumor immune response in tongue squamous cell carcinoma. Arch Oral Biol 92: 32-37. [Crossref]

14. Cheng L, Liu J, Liu Q, Liu Y, et al. (2017) exosomes from melatonin treated hepatocellular carcinoma cells alter the immunosuppression status through STAT3 pathway in macrophages. Int J Biol Sci 13: 723-734. [Crossref]

15. Zhang X, Zeng Y, Qu Q, Zhu J, Liu Z, et al (2017) PD-L1 induced by IFN-? from tumor-associated macrophages via the JAK/STAT3 and PI3K/AKT signaling pathways promoted progression of lung cancer. Int J Clin Oncol 22: 1026-1033.

16. Song X, Shao Y, Jiang T, Ding Y, Xu B, et al. (2018) radiotherapy upregulates programmed death ligand-1 through the pathways downstream of epidermal growth factor receptor in glioma. EBioMedicine 28: 105-113. [Crossref]

17. Wang Z, Jin H, Xu R, Mei Q, Fan D (2009) Triptolide downregulates Rac1 and the JAK/STAT3 pathway and inhibits colitis-related colon cancer progression. Exp Mol Med 41: 717-727.

Copyright: (C2019 Maruta H. This is an open-access article distributed under the terms of the Creative Commons Attribution License, which permits unrestricted use, distribution, and reproduction in any medium, provided the original author and source are credited. 